\title{
Estudio morfológico de diásporas de algunas especies de plantas acuáticas del Valle de México ${ }^{1}$
}

\author{
MARTHA OlVERA y ANTONIO LOT ${ }^{2}$
}

\begin{abstract}
RESUMEN. Se describe la morfología externa e interna de las diásporas de veintidos especies pertenecientes a doce familias de plantas acuáticas estrictas del Valle de México. Las características externas muestran gran variación a nivel de familia, mientras que en los taxa cercanamente relacionados se observa un patrón morfológico similar, y tanto la cubierta seminal como la superficie del pericarpo son de mayor valor taxonómico; la morfología interna de las semillas es muy semejante entre los taxa emparentados filogenéticamente. Se encontraron semillas albuminosas y exalbuminosas con dos tipos de embriones: amplio y linear, siendo este último el más común. El material de reserva de todas las semillas es almidón.
\end{abstract}

\begin{abstract}
External and internal morphology of diaspores of twenty two species of twelve families of aquatic plants of Valley of Mexico are described. External study of the seeds show remarkable variations among the families, while those taxa nearly related exhibit a similar morphological pattern. The seed coat and the surface of the pericarp show a high taxonomic value. The internal features of the seed are very similar within related taxa. There are two kinds of seed: 1) albuminous and 2) exalbuminous, and two embryo types, broad and linear; the linear embryo is more common among species. Starch is the food reserve in all seeds.
\end{abstract}

En general la información sobre la morfología de las diásporas de plantas vasculares acuáticas se encuentra en floras y revisiones taxonómicas, en las cuales frecuentemente son tratadas superficialmente. La morfología detallada de las diásporas proporciona descripciones útiles en las delimitaciones taxonómicas, sobre todo en las especies que

${ }^{1}$ Este trabajo representa una parte de la tesis de licenciatura presentada en 1988 por la primera autora en la Facultad de Ciencias, Universidad Nacional Autónoma de México.

2 Departamento de Botánica, Instituto de Biología, Universidad Nacional Autónoma de México, Apodo. Postal 70-233, Delegación Coyoacán, 04510, México, D. F. 
presentan alta plasticidad vegetativa como respuesta a cambios en el nivel del agua (de unos cuantos centímetros inclusive). Este fenómeno, por ejemplo, es común observarlo en Sagittaria latifolia que puede presentar hasta seis diferentes tipos de hojas dependiendo de las condiciones ambientales, conduciendo a determinaciones taxonómicas erróneas o a la derivación de variedades o subespecies cuando se trata sólo de especies polimorficas (Sculthorpe, 1967).

En este trabajo se describe la morfología de las diásporas de 22 de las especies de plantas acuáticas estrictas más conocidas de las 36 registradas en la literatura para el Valle de México, y se comentan algunas implicaciones taxonómicas y biológicas de sus caracteres morfológicos.

En este estudio se ha denominado diásporas a las semillas y a los frutos uniseminados, porque a menudo en las plantas acuáticas los pequeños frutos uniseminados una vez separados de la planta progenitora, resulta difícil diferenciarlos de las semillas.

\section{MATERIAL Y MÉTODO}

En la tabla 1 se presentan las especies estudiadas, las localidades de recolección y los recolectores. Las semillas y los frutos maduros se recolectaron en su habitat (diversas localidades de la Cuenca de México, excepto Ceratophyllum demersum que se recolectó en Campeche por no encontrarse material fértil en la región de estudio) o de ejemplares herborizados del Herbario Nacional (MEXU), Instituto de Biología, UNAM. El material se examinó con un microscopio estereoscópico y se hicieron diferentes mediciones de las diásporas para obtener el tamaño mínimo y máximo; el número de diásporas medidas dependió de la abundancia del material disponible, pero en promedio se hicieron observaciones de 20 diásporas (de varios individuos o ejemplares) de cada especie.

Para el estudio morfológico interno de la semilla, se realizaron secciones longitudinales a mano de las diásporas previamente hidratadas. Las secciones se tiñeron con una solución de yodo para identificar la región y tipo de material de reserva (Johansen, 1940). Sin embargo este procedimiento no permitió distinguir con claridad las estructuras, particularmente en las semillas pequeñas, por lo que se hicieron cortes más finos de la semilla por el método de inclusión en parafina. El material se escarificó e hidrató de 24 a $48 \mathrm{hr}$. a $4{ }^{\circ} \mathrm{C}$, se fijó en FAA (formol-alcohol-ácido acético), se deshidrató en series graduales de alcohol etílico y se incluyó en parafina (Johansen, 1940); se obtuvieron cortes longitudinales de 10 a $15 \mu$ en un microtomo de rotación y se tiñeron con safranina/verde-rápido (Sass, 1958). 


\section{RESULTADOS}

Morfología exterma. Las diásporas de los taxa estudiados son semillas y frutos uniseminados tales como mericarpos, aquenios y frutos parecidos a drupas y sus características morfológicas son resumidas en la tabla 2.

De manera general se observa entre las familias, que hay diferencias notables en forma, tamaño y cubiertas de las diásporas; mientras que a nivel interespecífico varían muy poco, aunque se aprecia un carácter constante entre los miembros de cada familia. Este hecho, por ejemplo, se observa claramente en las familias Pontederiaceae y Potamogetonaceae. En la primera las costillas longitudinales y las estrías transversales son características comunes entre las especies de Eichhornia y Heteranthera y, en la segunda el lomo dorsal de los frutos es un carácter común entre las especies de Potamogeton y Ruppia.

Asimismo, se nota que la morfología de la superficie de la cubierta seminal y del pericarpo, aporta diferencias significativas para la delimitación taxonómica de las especies. En el caso de las ninfáceas, se observaron 2 de los 4 tipos de cubierta seminal que Weberbauer (citado por Conard, 1905) distingue para la familia; uno de superficie glabra y costillas longitudinales (Nymphaea gracilis, fig. 1a) y el otro de superficie glabra, lisa y brillante (N. odorata, fig. $1 \mathrm{~b}$ ).

La superficie del pericarpo en Ceratophyllum también ofrece diferencias notables en las especies estudiadas. En $C$. demersum es lisa (fig. $1 \mathrm{k}$ ) y en $C$. muricatum es reticulada (figs. $2 \mathrm{a}, 2 \mathrm{~b}$ ).

Las semillas de las pontederiáceas estudiadas, muestran diferencias en la cubierta seminal, particularmente en la región calazal. Las semillas de Eichhornia tienen la región calazal truncada con una depresión circular de color obscuro (fig. 1g), mientras que en las de Heteranthera es mucronada (figs. 1h, 1i).

En contraste con lo anterior, en las semillas de Typha no se observaron diferencias notables. Tanto en $T$. domingensis como en T. latifolia la cubierta seminal es reticulada, la semilla de ambas especies son iguales en forma y hay sobreposición en su tamaño, siendo díficil separar las semillas de una y otra especie con base sólo en estas características.

Es importante señalar que la tipificación de los frutos no resultó fácil para todos los casos, particularmente en aquellos pertenecientes a la familia Potamogetonaceae. Estos han sido denominados por diferentes autores como drupeolas, nueces o aquenios, sin que ninguno de estos términos los describa adecuadamente. De acuerdo con Aalto (citado por Haynes, 1974) y nuestras observaciones, los frutos de potamogetonáceas son histológicamente drupas; están diferenciados en un exocarpo y un mericarpo carnosos y el endocarpo endurecido. Este último se abre a manera de tapadera a través de dos líneas a lo largo del lomo dorsal durante la germinación, por lo que son dehiscentes. Sin embargo, la drupas son frutos indehiscentes (Font Quer, 1965 y Radford, et al., 1974) y para diferenciar éstos de los frutos de las potamogetonáceas, se ha preferido usar en este trabajo el término "parecido a una drupa". Además, es importante subrayar que en 
TABLA 1. Especies de plantas acuáticas estudiadas

\begin{tabular}{|c|c|c|}
\hline TAXA & COLECTOR & LOCALIDAD \\
\hline $\begin{array}{l}\text { Sagittaria latifolia Willd. } \\
\text { var. latifolia }\end{array}$ & Lot y Novelo 898 & $\begin{array}{l}\text { Laguna de Texcaltengo al E de } \\
\text { Toluca, México }\end{array}$ \\
\hline S. macrophylla Zucc. & $\begin{array}{l}\text { B.V.A. } 650 \\
\text { Lot y Novelo } 1044 \\
\text { Olvera } 40\end{array}$ & $\begin{array}{l}\text { Laguna de Zumpango, Mun. } \\
\text { Zumpango, México } \\
\text { Canales aledaños a la Laguna de } \\
\text { Atenco al NW de Texcoco, México } \\
\text { Laguna Espejo de los Lirios, } \\
\text { Cuautitlán Izcalli }\end{array}$ \\
\hline Ceratophyllum dermersum L. & Ocaña 132 & $\begin{array}{l}\text { Laguna del Este, Mun. Cd. del } \\
\text { Carmen, Campeche }\end{array}$ \\
\hline C. muricatum Cham. & Olvera 10 & $\begin{array}{l}\text { Canales de Xochimilco, Distrito } \\
\text { Federal }\end{array}$ \\
\hline Triglochin mexicanum Kunth & $\begin{array}{l}\text { Rojas s/n } \\
\text { Lot y Novelo } 1188\end{array}$ & $\begin{array}{l}\text { Lago Cuitzeo, Michoacán } \\
\text { Llanos inundables a } 2.5 \mathrm{~km} \text { al E de } \\
\text { Tequiztla, Puebla }\end{array}$ \\
\hline $\begin{array}{l}\text { Lilaea scilloides (Poiret) } \\
\text { Hauman }\end{array}$ & $\begin{array}{l}\text { Lot y Novelo } 963 \\
\text { Novelo } 770\end{array}$ & $\begin{array}{l}\text { Desviación a Villa Alpina, carr. } \\
\text { México-Toluca } \\
\text { Charca a } 10 \mathrm{~km} \text { al NE de } \\
\text { Tepotzotlán, México }\end{array}$ \\
\hline Nymphoides fallax Ornduff & $\begin{array}{l}\text { Miranda } 153, \text { Olvera } 44 \\
\text { Olvera } 44\end{array}$ & $\begin{array}{l}\text { Laguna Espejo de los Lirios, } \\
\text { Cuautitlán-Izcalli }\end{array}$ \\
\hline Lemna gibba L. & $\begin{array}{l}\text { Barkley, Paxon \& } \\
\text { Webster } 2442\end{array}$ & Puebla \\
\hline $\begin{array}{l}\text { Najas guadalupensis (Sprengel) } \\
\text { Magnus var. guadalupensis }\end{array}$ & Lot y Novelo 1058 & $\begin{array}{l}\text { Zonas inundables al SE de la Presa } \\
\text { Cuevecillas, ca. autopista México- } \\
\text { Querétaro, México } \\
\text { Puerto Alegre, San Juan del Río, } \\
\text { Querétaro }\end{array}$ \\
\hline Nymphaөa gracilis Zucc. & $\begin{array}{l}\text { Lot y Novelo } 906 \text {, } \\
\text { Olvera } 45\end{array}$ & $\begin{array}{l}\text { Laguna Espejo de los Lirios, } \\
\text { Cuautitlán, Izcalli }\end{array}$ \\
\hline $\begin{array}{l}\text { N. odorata Ait. var. } \\
\text { gigantea Tricker }\end{array}$ & Lot y Novelo 1191 & $\begin{array}{l}\text { Lago Atezca, a } 6 \mathrm{~km} \text { al } \mathrm{E} \text { de } \\
\text { Molango, Hidalgo }\end{array}$ \\
\hline
\end{tabular}




\section{TAXA}

COLECTOR

LOCALIDAD

Eichhornia crassipes (C. Martius) Novelo s/n

Solms-Laub.

Heteranthera limosa (Sw.) Willd. Lot y Novelo 1178

Novelo 778

$s / 1$

H. peduncularis Benth

Novelo 768

Novelo 789

Charca a $10.2 \mathrm{~km}$ al $\mathrm{N}$ de

Tepotzotlán, México

Laguneta $10 \mathrm{~km}$ al $\mathrm{N}$ de Amealco,

Querétaro

Lot y Novelo 1987, 1177.

Charca al E de Tepotzotlán, México

Puerto Alegre, $10 \mathrm{~km}$ al $\mathrm{N}$ de

Amealco, Querétaro

Charca a $100 \mathrm{~m}$ de la Presa de la

Concepción, México

Potamogeton Illinoensis Morong

B.V.A. 672

Laguna de Tecocomulco, Hidalgo

P. nodosus Poiret

Novelo 769

Olvera 31

$10 \mathrm{~km}$ al NW de Tepotzotlán, México

Vivero municipal de Tepotzotlán, México

P. pectinatus L.

Olvera 12

Canales de Xochimilco, Distrito

Federal

Olvera 43

Exlago de Texcoco, México

P. pusillus L.

Olvera 1

Olvera 34

Laguna Guadalupe Victoria, México Vivero municipal de Tepotzotlán, México

Ruppia maritima L.

Olvera 42

Exlago de Texcoco, México

Typha dominguensis Presl

Lot y Novelo 1181

Laguna Espejo de los Lirios, Cuautitlán-Izcalli

T. latifolia L.

Miranda 2490

Lago de Epatlán, Puebla

Zannichellia palustris L.

Lot y Novelo 1038

Novelo 475

$1 \mathrm{~km}$ al E de la Planta Termoeléctrica del Valle de México, junto a

Totolcingo, México

Canales al $\mathrm{W}$ de la laguna de

Tecocomulco, Hidalgo

\footnotetext{
* explicación en el texto.
} 
TABLA 2. Características externas e internas de las diásporas estudiadas

$$
\text { Diáspora / Taxa }
$$

Forma

Tamaño

$(\mathrm{mm})$

\title{
Semilla
}

Nymphaøa gracilis (1)

N. odorata (1)

Nymphoides fallax (2)

Najas guadalupensis (3)

esferoide

$1.7 \times 1.4$

elipsoide

$1.8 \times 1.3$

elipsoide

$2.0 \times 1.6$

fusiforme

$2.6 \times 0.7$

Lemna gibba (4)

Typha domingensis (5)

T. latifolia (5)

ovoide a obovoide

$0.8 \times 0.6$

fusiforme

$0.8 \times 0.2$

fusiforme

$0.9 \times 0.2$

Eichhornia crassipes (6)

Heteranthera limosa (6)

oblongo obovoide

$1.5 \times 0.7$

elipsoide

$0.7 \times 0.3$

H. peduncularis (6)

elipsoide

$1.0 \times 0.3$

\section{Mericarpo}

Triglochin mexicanum (7)

linear triqueto

$4.1 \times 0.8$

\section{Aquenio}

Ceratophyllum demersum (8)

C. muricatum (8)

Sagittaria latifolia (9)

S. demersa (9)

Zannichellia palustris (10)

Lilaea scilloides (11)

elipsoide

elipsoide

obovoide

obovoide

obovoide

elipsoide

lanceolado triqueto

elipsoide
$4.5 \times 2.9$

$4.1 \times 2.6$

$3.5 \times 1.9$

$3.2 \times 1.4$

$2.0 \times 0.8$

$4.0 \times 2.0$

$4.5 \times 2.1$

\section{Parecido a drupa}

Potamogeton illinoensis (12)

$P$. nodosus (12)

$P$. pectinatus (12)

$P$. pusillus (12)

Ruppia maritima (12)

\author{
obovoide \\ obovoide \\ obovoide a orbicular \\ obovoide \\ ovoide
}

$2.2 \times 2.0$

$3.8 \times 2.7$

$2.6 \times 2.3$

$1.8 \times 1.6$

$1.8 \times 1.1$

(1) Nymphaeaceae, (2) Menyanthaceae, (3) Najadaceae, (4) Lemnaceae, (5) Typhaceae, (6) Pontederiaceae, (7) Juncaginaceae,

(8) Ceratophyllaceae, (9) Alismataceae, (10) Zannichelliaceae, (11) Lilaeaceae, (12) Potamogetonaceae. 


\begin{tabular}{|c|c|c|c|c|}
\hline & & orión & & Cubierta seminal \\
\hline Superficie y/o a & Tamaño & $\begin{array}{l}\text { No. de } \\
\text { cotiledones }\end{array}$ & $\begin{array}{l}\text { Endos- } \\
\text { permo }\end{array}$ & $\begin{array}{l}\text { No. de } \quad \text { Consistencia } \\
\text { capas }\end{array}$ \\
\hline
\end{tabular}

acostillada, arilo

lisa, arilo

areolada, aréolas irregulares

areolada, aréolas 4-6 ángulos

acostillada, opérculo prominente reticulada

reticulada

10-12 costillas, estriada

10-12 costrillas, estriada

8-10 costillas, estriada

acostillado dorsalmente

lisa, espinas 2 basales

tuberculada, espinas numerosas

laterales

alado, rostelo lateral

alado, rostelo subapical

espinoso lateralmente, estipitado

acostillado, alado

acostillado, rostelo tricorne

\begin{tabular}{|c|c|c|c|c|}
\hline$P$ & 2 & $t+$ & 2 & ext. papirácea \\
\hline $\mathrm{P}$ & 2 & $t+$ & 2 & int. esclerosada \\
\hline M & 2 & + & 1 & esclerosada \\
\hline $\mathrm{T}$ & 1 & - & 2 & $\begin{array}{l}\text { ext. esclerosada } \\
\text { int. membranosa }\end{array}$ \\
\hline T & 1 & - & 1 & esclerosada \\
\hline M & 1 & + & 2 & membranosa \\
\hline M & 1 & + & 2 & membranosa \\
\hline M & 1 & + & 1 & esclerosada \\
\hline$M$ & 1 & + & 1 & esclerosada \\
\hline M & 1 & + & 1 & esclerosada \\
\hline
\end{tabular}

T

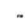

1

membranosa

T 2

T 2

$\begin{array}{ccc}\text { - } & 1 & \text { membranosa } \\ \text { - } & 1 & \text { membranosa } \\ \text { - } & 1 & \text { membranosa } \\ \text { - } & 1 & \text { membranosa } \\ \text { - } & 1 & \text { membranosa } \\ \text { - } & 1 & \text { membranosa }\end{array}$

2

1
1
1

a

lomo dorsal redondeado

lomo dorsal crestado

lomo dorsal liso

lorno dorsal redondeado

lomo dersal liso, caras

foveoladas

$T$
$T$
$T$

\footnotetext{
I - embrión indiferenciado, $M$ - mediano, $\mathrm{P}$ - pequeño, $\mathrm{T}$ - total, - (sin endospermo), + (abundante, ocupando la mitad de la cavidad de la semilla), ++ (endosepermo escaso, perispermo muy abundante).
} 
Potamogeton se describe la morfología del exocarpo, mientras que en Ruppia se describe la del endocarpo, ya que las partes carnosas en esta última son muy delgadas y se desprenden tempranamente dejando expuesto el endocarpo.

Morfología interna. Con base en la clasificación de los embriones propuesta por Martin (1946), se encontraron básicamente 2 tipos de semillas. En primer lugar, las semillas con el embrión de tipo amplio, presente en las especies de Nymphaea. Este embrión ocupa un volumen pequeño en la región micropilar rodeado de unas cuantas células del endospermo y embebido en el tejido de reserva o perispermo (Conard, 1905 y Wiersema, 1987) que contiene almidón.

En segundo término, las semillas con el embrión de tipo linear, que se observó en 13 de los 14 géneros estudiados. Los embriones generalmente son más largos que anchos, rectos, curvados o doblados; de tamaño mediano a total y diferenciados o no (fig. 3a-m). Martin (1946) expresa el tamaño del embrión en proporción de su volumen y la cantidad de endospermo, de tal manera que las semillas se subdividen en: a) exalbuminosas con el embrión total que ocupa toda la cavidad de la semilla y b) albuminosas con el embrión mediano. En este último, el embrión ocupa la mitad de la cavidad de la semilla y se encuentra rodeado por el endospermo, como se observa en las especies de Nymphoides, Typha, Eichhornia y Heteranthera, que también contiene almidón.

En la mayoría de las especies de las monocotiledóneas (véase tabla 2) el embrión no está diferenciado, excepto en Ruppia maritima y en Zannichellia palustris en las que se observa un cotiledón y un hipocótilo macrópodo, además de la radícula en $R$. maritima (figs. 3e, 3f).

En contraste, en las semillas de la dicotiledóneas observadas (Nymphaea, Nymphoides y Ceratophyllum) el embrión está diferenciado en cotiledones, hipocótilo y/o epicótilo y/o radícula. Además se observa una alta diferenciación de la plúmula en Ceratophyllum. En C. demersum la primera hoja de la plúmula es simple, mientras que en $C$. muricatum (fig. 31) es generalmente bipartida y ocasionalmente simple.

La cubierta seminal en los taxa con mericarpos (Triglochin), aquenios (Ceratophyllum, Sagittaria, Zannnichellia y Lilaea) y frutos parecidos a drupas (Potamogeton y Ruppia), está reducida o modificada a una capa membranosa, pero de cierta resistencia. Por el contrario, en los taxa con semillas como unidad de dispersión (véase tabla 2), la cubierta seminal está esclerosada y su extraordinaria dureza se debe a la paredes celulares lignificadas de la cubierta. 

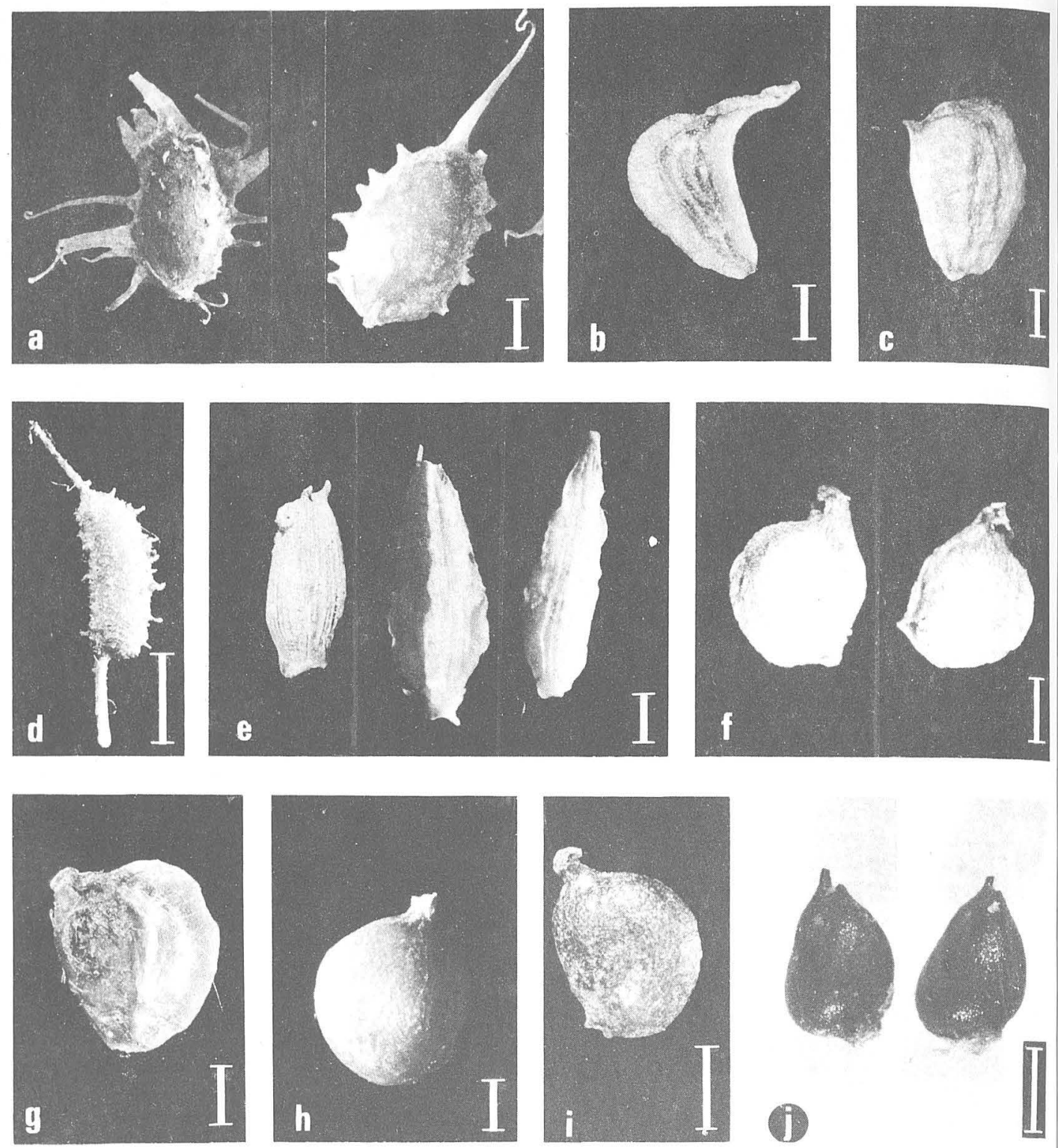

Fig. 2. a-e aquenios. a. Ceratophyllum muricatum (Olvera 10); b. Sagittaria latifolia (Novelo s/n); c. S. macrophylla (Lot \& Novelo 1044); d. Zannichellia palustris (Novelo 475); e. Lilaea scilloides (Lot \& Novelo 963); f-j. drupas, f. Potamogeton illinoensis (BVA 672); g. P. nodosus (Novelo 769); h. P. pectinatus (Olvera 43); i. P. pusillus (Olvera 1); j. Ruppia maritima (Olvera 42). Escala $=1 \mathrm{~mm}$. 

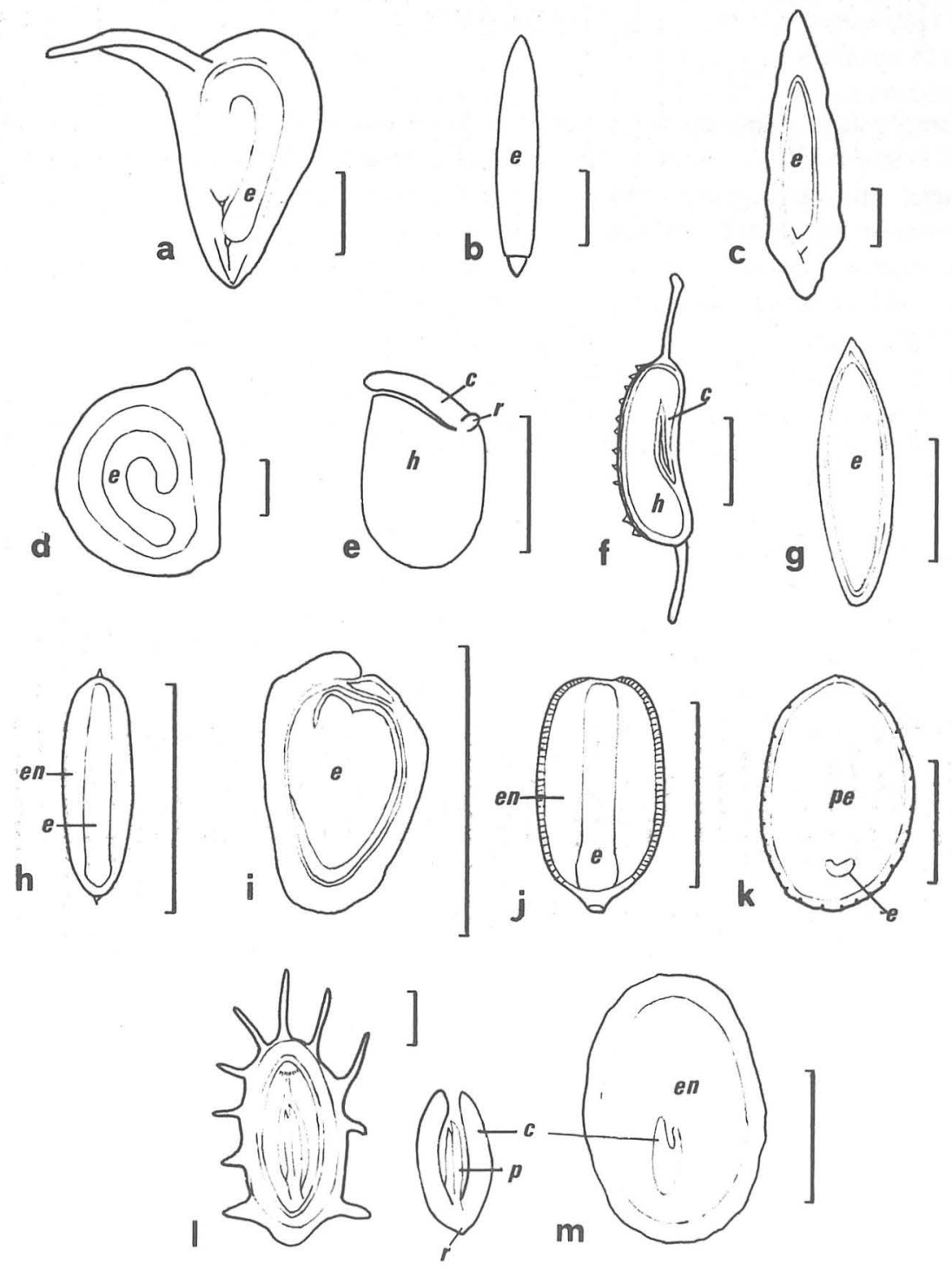

Fig. 3. a-m cortes longitudinales de frutos y semillas. a. Sagittaria latifolia; b. Triglochin mexicanum; c. Lilaea scilloides; d. Potamogeton pectinatus; e. Ruppia maritima; f. Zannichellia palustris; g. Najas guadalupensis; h. Typha domingensis; i. Lemna gibba; j. Eichhornia crassipes; k. Nymphaea odorata; 1. Ceratophvllum muricatum; m. Nymphoides fallax. c (cotileđón), e (embrión), en (endospermo), h (hipocótilo), p (plúmula), pe (perispermo), $\mathbf{r}$ (radícula). Escala $=1 \mathrm{~mm}$. 


\section{DisCUSIÓN}

Como puede verse en los resultados, la variación morfológica externa de las diásporas se manifiesta en la forma, el tamaño, la superficie de la cubierta y en los apéndices como alas, espinas, costillas, etc. Esta variación ofreció caracteres confiables en la caracterización de las hidrofitas aquí tratadas, por lo que tienen valor taxonómico para separar las especies, lo que concuerda con lo señalado por Lawrence (1951) y David y Heywood (1963) para las plantas vasculares en general.

Los resultados muestran que en la mayoría de los taxa la delimitación de las especies se basa en la superficie de la semilla o del pericarpo, lo que sugiere que este carácter tiene mayor valor taxonómico que el resto de los caracteres.

Lo anterior se confirma con lo observado en las semillas de las ninfáceas, en donde la cubierta seminal proporcionó diferencias significativas entre Nymphaea gracilis y $N$. odorata. De hecho, Wiersema (1987) reconoce el valor sistemático de este carácter en las ninfáceas y distingue 4 tipos de cubierta seminal para las especies del subgénero Hydrocallis. De igual manera, la importancia taxonómica de la cubierta seminal es considerada en Najas. Haynes (1977) menciona que los caracteres de la semilla son los más importantes para distinguir muchas de las especies de este género.

Para el caso de las semillas de Typha, en las cuales la cubierta seminal no mostró diferencias apreciables entre T. domingensis y T. latifolia, indica el poco valor taxonómico de este carácter en estas especies.

Con respecto a la problemática de la tipificación de los frutos de las plantas acuáticas, se señaló anteriormente que la clasificación de los frutos propuesta por Radford, et al. (1974), basada en el origen, textura y dehiscencia de los frutos, en muchos casos no es útil al intentar definir los frutos de estas plantas. Dalgren y Clifford (1982) mencionan que particularmente en las monocotiledóneas esta terminología es muy complicada y no es fácil interpretar las descripciones que hacen de éstos. Particularmente en las plantas acuáticas con frutos sumergidos, como en Potamogeton, Najas, Ruppia, Ceratophyllum, etc., el uso de la palabra "parecido a" precedido del tipo de fruto puede ser recomendado para indicar cierta variación en estos frutos que indiquen alguna adaptación al medio acuático.

En lo que respecta a la morfología interna de la semilla, Martin (1946) señala que ésta es constante en los taxa cercanamente relacionados pudiéndose agrupar en categorias taxonómicas superiores a la de familia y aporta información importante para explicar sus relaciones de parentesco.

Los resultados de este trabajo muestran en efecto, que las características del embrión son muy constantes dentro de los taxa cercanamente emparentados. Así, el embrión pequeño de tipo amplio observado en las especies de Nymphaea, se presenta además en los miembros del orden Nymphaeiflorae (Dahlgren y Clifford, 1982). El tipo de embrión observado en las tifáles también se presenta en el superorden Commeliniiflorae y el de las pontederiáceas en el superorden Liliiflorae (Dalhgren y Clifford, 
1982). El resto de las especies estudiadas, excepto Lemna y Ceratophyllum, con el embrión linear, de tamaño total y sin endospermo pertenecen al superorden Alismatiflorae (Dahlgren y Clifford, 1982). Asimismo, este grupo es incluido en el orden Helobiae (Alismatidae) según la clasificación de Tomlinson (1982), quien menciona que la falta de endospermo en la semilla de estos taxa, se debe a que durante la maduración de la semilla el endospermo es reabsorbido por el embrión.

En cuanto a la presencia de embriones macrópodos, Dahlgren y Clifford (1982) los han reportado sólo en las monocotiledóneas, concretamente en Najadales (Najas) y Zosterales (Ruppia y Zannichellia, entre otros). Sin embargo, en Najas guadalupensis no se observó el embrión diferenciado, probablemente debido a que el material estudiado estaba en un estado temprano de desarrollo.

Con respecto a la diferenciación del embrión en las dicotiledóneas, de particular interés es la alta diferenciación de la plúmula observada en el embrión de Ceratophyllum. Esta característica ha sido considerada de importancia taxonómica por Muenscher (1940) y Les (1985) para separar las diferentes especies de Ceratophyllum dado el polimorfismo vegetativo y de los frutos. Sin embargo, Lowden (1978) cuestiona la validez taxonómica de este carácter ya que él observó en algunas semillas de $C$. muricatum la primera hoja de la plúmula simple como en $C$. demersum, observaciones que coinciden con las nuestras.

Otro aspecto interesante de la morfologia de las semillas, es la constitución de la cubierta seminal. La reducción de ésta en los frutos indehiscentes, se explica en parte por la presencia de pericarpos esclerosados, como los de Ceratophyllum, o bien por la presencia de pericarpos delgados pero resistentes e impermeables como los de Sagittaria, en los cuales la función protectora de la cubierta seminal es sustituida por el pericarpo.

La presencia de cubiertas seminales o pericarpos esclerosados (en los frutos uniseminados), como ya ha sido mencionado por Arber (1972), Croker (1907) y Sculthorpe (1967), explica el porqué la mayoría de las semillas de las hidrofitas presentan una latencia prolongada y no ocurre la germinación hasta que la pared del pericarpo se pierde o bien hasta que el micrópilo pierde la capa cutinizada que lo protege y, por otra parte, también explica la tolerancia de las diásporas a una prolongada inmersión.

AgRADECIMIENTOS. Agradecemos a la Dra. Judith Márquez las facilidades prestadas en la realización del trabajo de laboratorio y al M. en C. Alejandro Martínez por el trabajo fotográfico, ambos de la Facultad de Ciencias, UNAM. A la Dra. Patricia Dávila del Instituto de Biología, UNAM, la revisión crítica del manuscrito. 


\section{LITERATURA CITADA}

ARBER, A. 1972. Water plants. A study of aquatic angiosperms. Cambridge University Press. 436p.

CONARD, H.S. 1905. The waterlilies: a monograph of genus Nymphaea. Publ. Carnegie Inst. Wash. 4:1-279

CROKER, W. 1907. Germination of seeds of water plants. Bot. Gaz. (Crawfordville). 44:375-380.

DAHLGREN, R.M. y H.T. ClifFORD. 1982. The monocotyledons: a comparative study. Academic Press. Inc. 378p.

DAVIS, P.H. y V.H. HEYWOOD. 1963. Principles of angiosperm taxonomy. Robert E. Krieger Publish. Company. Huntington, New York. 550p.

FONT QUER, P. 1965. Diccionario de botánica. Editorial Labor, Barcelona. 1244p.

HAYNES, R.R. 1974. Revision of the North American Potamogeton subsection pusilli (Potamogetonaceae). Rhodora 76(808):564-649.

HAYNES, R.R. 1977. The Najadaceae in the southeastern United States. J. Arnold Arbor. 58(2):161-170.

JOHANSEN, A.D. 1940. Plant microtechnique. McGraw Hill, New York. 523p.

LAWRENCE, G.H.M. 1951. Taxonomy of vascular plants. MacMillan Pub. Co., Inc. New York. 823p.

LES, D.H. 1985. The taxonomic significance of plumule morphology in Ceratophyllum (Ceratophyllaceae). Syst. Bot. 10(3):338-346.

LOWDEN, R.M. 1978. Studies on the submerged Ceratophyllum L. in the neotropics. Aquat. Bot. 4(2):127-142.

MARTIN, A.C. 1946. The comparative internal morphology of seed. Amer. Midl. Naturalist 36:513-660.

MUENSCHER, W.C. 1940. Fruits and seedlings of Ceratophyllum. Amer. J. Bot. 27:231.

RADFORD, A., W.C. DiCKInSON, J.R. MASSEY y C.R. BELL. 1974. Vascular plant systematics. Harper \& Row, New York. 891p.

SASS, J.E. 1958. Botanical microtechnique and cytochemistry. 3a. ed. The Iowa State University, Iowa. 228p.

SCULTHORPE, C.D. 1967. The biology of aquatic vascular plants. Edward Arnold, London. 610p.

Tomlinson, P.B. 1982. Anatomy of the monocotyledons. VII. Helobiae. Ed. C.R. Metcalfe. Clarendon Press, Oxford. 551p.

WIERSEMA, J.H. 1987. A monograph of Nymphaea subgenus Hydrocallis (Nymphaeaceae). Syst. Bot. Monogr. 16:1-112. 